\title{
100 Gbit/s Real-Time All-Analogue Filter Bank OFDM based on a Gain-Switched Optical Comb
}

\author{
F. A. Gutiérrez ${ }^{(1)}$, E. P. Martin ${ }^{(1)}$, P. Perry ${ }^{(1)}$, A. D. Ellis ${ }^{(2)}$, P. Anandarajah ${ }^{(1)}$, F. Smyth ${ }^{(1,3)}$, L. P. Barry ${ }^{(1)}$ \\ (1) The RINCE Institute, Dublin City University, Glasnevin, Dublin 9, Ireland, fernando.gutierrez@dcu.ie \\ (2) Aston Institute of Photonic Technologies, Aston University, Aston Triangle, Birmingham B4 7ET, UK \\ (3) Pilot Photonics, Invent Centre, Dublin City University, Glasnevin, Dublin 9, Ireland.
}

\begin{abstract}
A real-time 5x21.6 Gbit/s WDM electro-optical transceiver is presented. Optical carriers were spaced by $20 \mathrm{GHz}$ and each one transmitted four orthogonally overlapping broadband subcarriers. Only analogue electronics were employed, achieving an unprecedented spectral efficiency in DSPless SCM links.
\end{abstract}

\section{Introduction}

The increasing demand for capacity in optical networks has translated into a growing interest and research effort in high-speed optical interconnects and its associated technologies ${ }^{1}$. In recent years, the majority of advances in the area have been supported by digital signal processing (DSP) due to its capacity to increase spectral efficiency and mitigate impairments. However, a high number of computational operations also brings about disadvantages such as increased power consumption ${ }^{2}$ and often latency. In contrast, many intra data centre subsystems need moderate to high capacity while reducing power consumption and latency to a minimum. For such applications, a purely analogue implementation presents the best trade-off between those parameters, especially if orthogonal frequency division multiplexing (OFDM) can be achieved. Although this concept has already been envisaged and simulated $^{3}$, no physical realisation has yet been reported.

This work presents a $100 \mathrm{Gbit} / \mathrm{s}$ multichannel direct detection (DD) OFDM electro-optical transceiver where only off-the-shelf microwave components are used to orthogonally modulate and demodulate the transmitted data. The bit rate of the baseband signals is selected to be 2.7 Gbit/s, which is compatible with the operative frequency of central processing units (CPU) and random access memory (RAM) interfaces. Potentially, new system on chip (SoC) DSP-less architectures can be implemented achieving lower latency and power consumption while meeting specific spectral efficiency requirements.

\section{Filter Bank Multicarrier (FBMC)}

$\mathrm{FBMC}^{4}$ is a scheme that allows the transmission of orthogonal subcarriers without using the fast Fourier transform (FFT). Three conditions must be met: baseband transmitted and received data must be filtered with the same Nyquist roll-off; for every subcarrier, in-phase and quadrature baseband data must be delayed half a bit period with respect to each other; and, finally, local oscillators (LO) must be delayed half pi radians alternately. This technique allows an allanalogue implementation and was employed in this work. Although microwave Nyquist filters with constant group delay are impossible to build, a pseudo ideal solution can be found employing standard low pass filters (LPF) ${ }^{3}$. Unlike FFT-based OFDM, with this method the dispersion suffered by any electrical subcarrier will only influence the performance of its adjacent subchannels. For low transmission distances, this penalty would be negligible while the absence of a cyclic prefix maximises the data rate.

\section{Experiment}

The setup used in the experiment is illustrated in Fig. 1. The electrical scheme was equivalent to a traditional quadrature phase-shift keyed (QPSK) single side band (SSB) subcarrier multiplexing (SCM) system, but incorporating orthogonally overlapping subcarriers and the conditions explained in the previous section. For the low pass orthogonality filters, a particular combination of vendor-specific and Bessel filters was employed. Eight uncorrelated $2.7 \mathrm{Gbit} / \mathrm{s}$ data streams were generated. The four pairs of data signals were used to modulate four local oscillators (LO) located at the harmonics of the data rate (from 5.4 to $13.5 \mathrm{GHz}$ inclusive). The QPSK modulation was performed using four low cost off-the-shelf monolithic microwave integrated circuit (MMIC) IQ mixers. These devices are designed for radio transmission and do not have the group delay optimised for broadband communications. Hence, pseudo random binary sequences (PRBS) of 127 bits were transmitted. The outputs of the $I Q$ mixers were combined, generating a $21.6 \mathrm{Gbit} / \mathrm{s}$ OFDM signal, and fed to a $90^{\circ}$ hybrid splitter that 


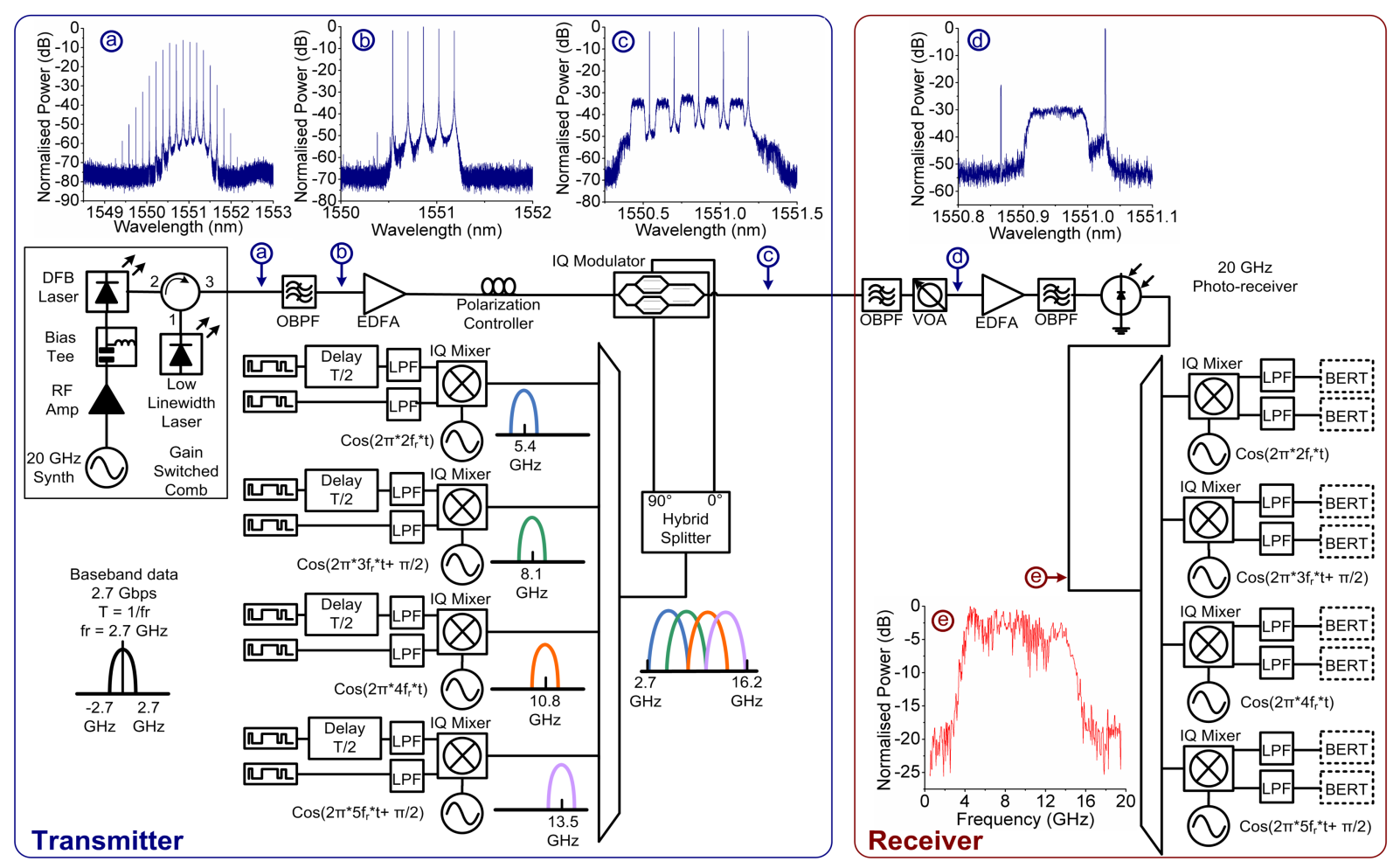

Fig. 1: 5x21.6 Gbit/s All-analogue OFDM electro-optical transceiver with a gain switched comb.

performed the Hilbert Transform (HT) required to achieve SSB. The electrical receiver performed the equivalent demodulation with the same family of IQ mixers. The obtained baseband signals were fed to a bit error rate tester (BERT).

To achieve wavelength division multiplexing (WDM), an externally injected gain-switched optical comb source ${ }^{5}$ with a free spectral range (FSR) of $20 \mathrm{GHz}$ was employed (Fig. 1, inset (a)). Five optical carriers were filtered (Fig. 1, inset (b)) and then amplified with an Erbium doped fibre amplifier (EDFA). The resultant comb was optically modulated emulating a WDM 5x21.6 Gbit/s scheme (Fig. 1, inset (c)). An optical IQ modulator, biased at quadrature, was employed to generate the optical lower SSB signal $^{6}$. An rms optical modulation index of $20 \%$ achieved a good trade-off between the carrier to signal ratio and the nonlinearities generated by the optical modulator ${ }^{6}$. The desired optical channel was then selected with a tunable optical band pass filter (OBPF) (Fig. 1, inset (d)). The rejection ratio of the adjacent optical carrier was $22 \mathrm{~dB}$. A variable optical attenuator (VOA) was used to simulate fibre losses. The resultant signal was introduced into a pre-amplified receiver consisting of an EDFA and a photoreceiver. The EDFA was operated in constant power mode to ensure an average optical power of $5 \mathrm{dBm}$ was fed to the photo-receiver. The electrical OFDM signal was obtained at the output of the photo-receiver (Fig. 1, inset (e)), and then demodulated.

With this close allocation of optical channels there are two contributions that set the minimum distortion of the system. Firstly, in the generation of the SSB signal, a suppressed sideband ratio of $20 \mathrm{~dB}$ was achieved. Therefore, the imperfectly suppressed side band from the neighbouring optical channel distorted the desired subcarriers ${ }^{7}$. Secondly, as can be seen from Fig. 1, inset (d), the optical carrier of the adjacent optical channel could not be perfectly suppressed in the receiver, adding cross-talk due to the beating in the photo-detector. As the transmitted baseband streams are uncorrelated, and with the selected frequency plan, it can be deduced that both distortions are uncorrelated with the desired signal. Therefore, no further decorrelation of the WDM signal is required.

\section{Results}

The performance of the system was measured obtaining the bit error rate (BER) as a function of the average optical power $P_{I N}$ at the input of the receiver EDFA. As it can be seen from Fig. 1, inset (b), the worst case optical channel was the fifth one, as it had the lowest optical carrier to noise ratio of the comb lines. Fig. 2 shows its BER measured for the four subcarriers (averaging the in-phase and quadrature components). However, a more realistic 
measurement of performance requires individually analysing the 40 subchannels (5x4x2, 5 optical carriers, 4 electrical subcarriers, and 2 due to the quadrature modulation on each subcarrier). The sensitivity of each subchannel, considering a $7 \%$ forward error correction (FEC) code, is illustrated in Fig. 3 . The worst case is a subchannel of the first subcarrier within the fifth optical channel, with a sensitivity of $-20.5 \mathrm{dBm}$. This performance is attributed to the subchannel being affected by the highest optical noise from the comb and the highest second order intermodulation distortion due to the beating at the photo-receiver ${ }^{6}$. Different performance between subchannels can also be attributed to the amplitude asymmetry of the optical carriers (Fig 1, inset (b)), and the varying behaviour of the IQ mixers in each of the frequency bands. After FEC overheads, the net data rate of $100 \mathrm{Gbit} / \mathrm{s}$ was achieved.

Discussion on the results and the technique Traditional broadband SCM links have been implemented using BPSK and QPSK. Higher order modulation formats have required $\mathrm{DSP}^{7}$. In the QPSK case, neglecting the optical carrier and considering only SSB realisations, spectral efficiency is $1 \mathrm{bit} / \mathrm{s} / \mathrm{Hz}^{7}$. In the same conditions, the scheme presented in this work achieves a spectral efficiency of $1.6 \mathrm{bit} / \mathrm{s} / \mathrm{Hz}(21.6 \mathrm{Gbit} / \mathrm{s}$ in $13.5 \mathrm{GHz})$, tending to 2 with a higher number of

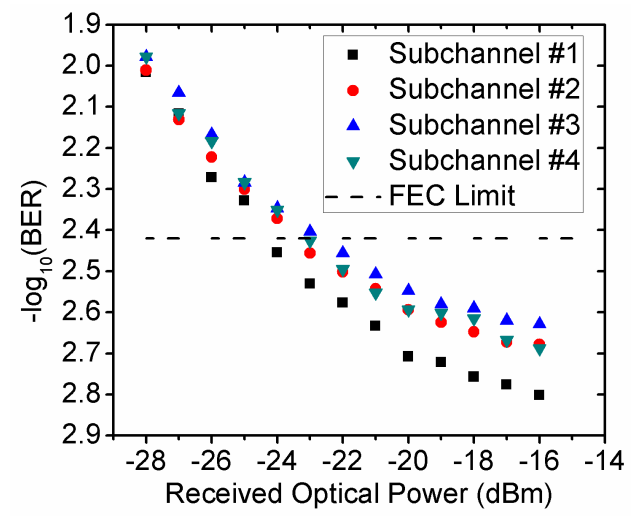

Fig. 2: Optical channel 5 performance.

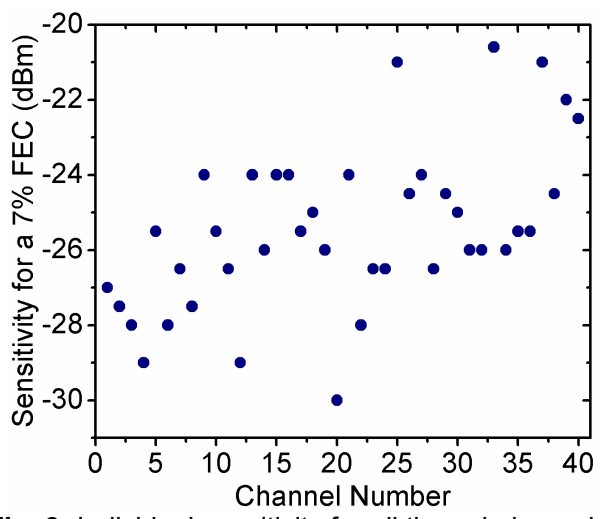

Fig. 3: Individual sensitivity for all the subchannels. subcarriers. All-analogue SCM links can benefit from this increment in performance while conserving a single comparator in the baseband receiver interface.

The proposed analogue OFDM system can be potentially applied to longer transmission distances. Coherent WDM presents similar properties and dispersion penalties are small even at high bit rates $^{8}$. Moreover, from a theoretical perspective, the presented OFDM 2.7 Gbaud SCM technique fits within the range of optimum subcarrier spacing ${ }^{9}$.

\section{Conclusions}

A WDM/OFDM 100 Gbit/s electro-optical transceiver based on a gain switched comb has been demonstrated. The novelty resides in an analogue implementation where inexpensive microwave IQ mixers and low pass filters permit the orthogonal transmission. An unprecedented spectral efficiency in purely analogue DD/SCM links has been achieved, with $1.6 \mathrm{bit} / \mathrm{s} / \mathrm{Hz}$ in the electrical signal and $>1 \mathrm{bit} / \mathrm{s} / \mathrm{Hz}$ including the pilot carriers and guard bands.

\section{Acknowledgements}

This work has been supported in part by Enterprise Ireland (CF/2011/1627) and Science Foundation Ireland (12/RC/2276).

\section{References}

[1] Z. Li et al., "Optical Interconnects: recent advances and future challenges," Optics Express, Vol. 23, no. 3, p. 3717 (2015).

[2] A. Morea et al., "Power Management of Optoelectronics Interfaces for Dynamic Optical Networks," Proc. ECOC, We.8.K.3, Geneva (2011).

[3] K.P. Benterud et al., "Quasi-orthogonal Subcarrier Multiplexing for high-capacity optical data links," Canadian Journal of Electrical and Computer Engineering, vol. 18, no. 4, p. 159 (1993).

[4] B. Farhang-Boroujeny, "OFDM versus Filter Bank Multicarrier," IEEE Signal Processing Magazine, Vol. 28, no. 3, p. 92 (2011)

[5] P. Anandarajah et al., "Flexible Optical Comb Source for Super Channel Systems," Proc. OFC, OTH3I.8, Anaheim (2013).

[6] F. A. Gutiérrez et al, "Optimum Bias Point in Broadband Subcarrier Multiplexing with Optical IQ Modulators," J. Lightwave Technology, vol. 33, no. 1, p. 258 (2015).

[7] F. A. Gutiérrez et al, "Impact of Band Rejection in Multichannel Broadband Subcarrier Multiplexing," J. Optical Comms. and Netw., vol. 7, no. 4, p. 248 (2015).

[8] F. C. G. Gunning et al, "Dispersion Tolerance of Coherent WDM," IEEE Phot. Techn. Letters, vol. 18, no. 12, p. 1338 (2006)

[9] P. Poggiolini et al., "Analytical results on system maximum reach increase through symbol rate optimization," Proc. OFC, Th3D.6, Los Angeles (2015). 